\title{
Flow Stress Characteristics of AZ31B Magnesium Alloy Sheet at Elevated Temperatures
}

\author{
Li Wen-Juan, Zhao Guo-Qun, Ma Xin-Wu, and Gao Jun
}

\begin{abstract}
The true stress-strain curves of AZ31B magnesium alloy sheet were obtained by using the uniaxial tensile tests at temperatures ranging from $50^{\circ} \mathrm{C}$ to $300^{\circ} \mathrm{C}$ and initial strain rates ranging from $0.001 / \mathrm{s}$ to $0.1 / \mathrm{s}$. The effects of temperature and strain rate on the flow stress were analyzed. The results show that the flow stress decreases and the elongation at fracture increases with increasing temperature and decreasing strain rate, and the formability is improved obviously. Two mathematical models were developed to determine the flow stress at hot deformation condition:(1) the model based on the Fields-Backofen equation, and (2) the model based on a natural exponential form whose exponent is a quadratic function. By comparison of the calculated results with experimental data, it was proved that the flow stresses calculated by the Fields-Backofen model only give a good fit to the measured ones at the work-hardening stage, but it cannot describe the softening behavior after the peak stress. Whereas, the exponential model does not only accurately reflect work hardening before the peak stress,but approaches more closely at the dynamic softening stage of the flow stress than that of the Fields-Backofen model.
\end{abstract}

Index Terms-AZ31B magnesium alloy, uniaxial tensile, dynamic recrystallization (DRX), flow stress, mathematical model.

\section{INTRODUCTION}

In recent years, magnesium alloys have received more attention in the transportation, aerospace and electronic communication industries due to their excellent physical and mechanical properties such as low density, high specific strength and specific rigidity, strong electromagnetic shielding, good thermal conductivity and recyclability [1]. At present, the majority of structural magnesium alloy products are still fabricated by die-casting. But as-casted alloy provides low mechanical strength due to the presence of casting defects such as inclusions, interdendritic shrinkage voids and porosity [2]. In contrast, wrought magnesium alloys have higher strength, better ductility and more varied mechanical properties as a result of improved microstructure control and optimized heat treatment, and have a broader prospect of market development [3], [4].

Magnesium alloys usually exhibit limited ductility at room temperature because of their hexagonal close-packed (HCP) crystal structure, which greatly restricts their

Manuscript received February 29, 2012; revised March 29, 2012.

W.-J. Li, G.-Q. Zhao, and X.-W. Ma are with Key Laboratory for Liquid-Solid Structural Evolution and Processing of Materials (Ministry of Education), Shandong University, Shandong, Jinan 250061, China, (email: liwj@sdu.edu.cn, zhaogq@sdu.edu.cn, maxinwu@sdu.edu.cn).

J. GAO is with School of Mechanical, Electrical and information Engineering, Shandong University at Weihai, Shandong, Weihai 264209, China (email: dshdgj@sdu.edu.cn). application scope in plastic forming fields. Fortunately, the plastic formability of magnesium alloys can be improved dramatically with the increase in temperature, which can be attributed to the activation of non-basal slips and the occurrence of dynamic recrystallization (DRX) at elevated temperatures [5]. It is reported that the majority of wrought magnesium alloys have good formability at high temperatures between $150^{\circ} \mathrm{C}$ and $300^{\circ} \mathrm{C}$ [6]. From the existing literatures, the development of hot rolling and extrusion process for magnesium alloys is relatively mature and they have been used in actual production, but the study of stamping process for magnesium alloy sheet is still mainly in the experimental stage. It is an urgent need to reduce production costs, increase productivity and improve product quality for the stamping process of magnesium alloy sheet. The flow stress at high temperatures is one of the indispensable information for evaluating the stamping performance of magnesium alloy sheet. The equation for flow stress is also the preliminary requirement for an accurate simulation of stamping process. As for stamping process, AZ31 magnesium alloy (aluminum 3\%, zinc 1\%) sheet are commercially produced and most commonly used.

Many flow stress models have been proposed, which can be divided into the following three types: (1) The first type of model is built based on Fields-Backofen equation [7], [8]. This model contains many non-independent parameters, which will make regression analysis more complex and the results inaccurate; (2) The second type of model is built by using the Arrhenius equation and introducing the Zener Hollomon parameters [9], [10].This model is mainly used for the prediction of steady-state flow stress, and much suitable for axial compression; (3) The third is an artificial neural network model introduced [11]. The neural network model has higher fitting precision. But it is hard to be implemented into commercial finite element software, and so its application is restricted.

In this study, the uniaxial tensile tests of AZ31B magnesium alloy sheet were conducted at temperatures ranging from $50{ }^{\circ} \mathrm{C}$ to $300{ }^{\circ} \mathrm{C}$ and initial strain rates of $0.001 / \mathrm{s}, 0.01 / \mathrm{s}$ and $0.1 / \mathrm{s}$. A group of true stress- strain curves were obtained. The effects of temperature and strain rate on flow stress were analyzed. Through the analysis of the true stress-strain curves,two mathematical models of flow stress were established.

\section{MATERIALS AND METHODS}

The material used in this study was a commercial magnesium based alloy AZ31B cross-rolled sheet with a thickness of $0.8 \mathrm{~mm}$, whose chemical composition was (wt-\%) Mg-2.79Al-0.99Zn-0.36Mn.The as-received material, whose microstructure is shown in Fig. 1, has 
homogeneous equiaxial fine grains, with the average grain size of about $15 \mu \mathrm{m}$.

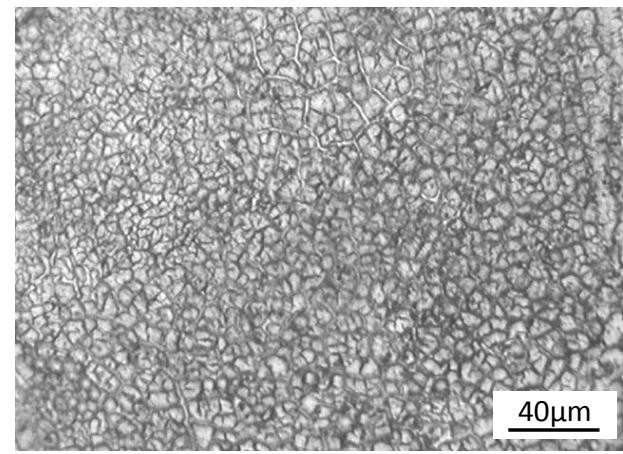

Fig. 1. Microstructure of AZ31B magnesium alloy sheet.

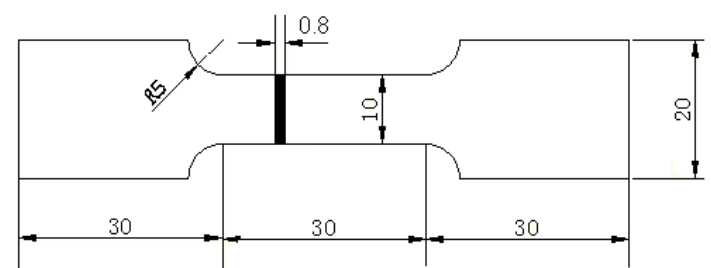

Fig. 2. Shape and dimensions of tensile specimens(mm)

The specimens were electro-discharged machined from the sheets with the tensile axis parallel to the finial rolling direction. Fig. 2 shows the geometries of the specimens. The hot tensile tests were conducted on Gleeble-1500D thermo-simulation machine at temperatures ranging from $50{ }^{\circ} \mathrm{C}$ to $300{ }^{\circ} \mathrm{C}$ and initial strain rates of $0.001,0.01$ and 0.1 /s. The specimens were rapidly heated by resistance heating and the temperature was controlled and measured by a thermocouple spot welded to the surface of the specimen at the mid-height. The tests were carried out in the vacuum chamber to minimize the high-temperature oxidation of the specimens. The load- displacement data for every tensile test were recorded automatically by the computer control system of the thermal simulator and processed to obtain true stress-strain curves using standard equations. Fig. 2 shows tensile specimens both before and after testing. It was observed that, at low temperatures, the fracture occurs by brittle cleavage; however the fracture occurs by ductile rupture at high temperatures above $200^{\circ} \mathrm{C}$ with an obvious necking phenomenon.

\section{ANALYTICAL RESUltS AND DisCUSSION}

The true stress-strain curves of AZ31B magnesium alloy at various temperatures and strain rates are shown in Fig. 4. For temperatures lower than $150{ }^{\circ} \mathrm{C}$, the flow stress rises constantly with increasing strain and the curve only exhibits work hardening and/or stable stage. Whereas, for temperatures higher than $200{ }^{\circ} \mathrm{C}$, the flow stress curves can be divided into three stages, namely hardening, stable and softening stages[10]. Initially the stress rises abruptly with strain then increases at decreased rate up to peak stress, which indicates that work hardening plays a dominant role in the initial stage of deformation. The presence of a peak in the flow stress curve reveals the beginning of DRX. When the strain exceeds a critical value, the flow stress begins to decrease with increasing strain until the end of the test as softening caused by DRX overtakes hardening caused by work hardening [8]. At higher temperatures such as $300^{\circ} \mathrm{C}$, the flow stress curve exhibits much gentler softening after the peak and the peak stress is almost the same as its correspondent flow stress, which indicates that there is a dynamic balance between work hardening and softening [8]. It is also found in Fig. 4 that the peak stress decreases with increasing temperature and/or decreasing strain rate, and the work hardening rate is significantly reduced before the peak stress, while the softening stage becomes longer after the peak stress. This is because the increase in temperature will increases atomic activity, the work hardening induced by the dislocation pile-up is significantly weakened, and the strain softening effect is strengthened. The strain rate dependence of flow stress can be explained as follows: the increase of strain rate will accelerate dislocation pile-up, which needs a higher shear stress, and thus the flow stress increases. In addition, when the deformation speed increases, the material does not have enough time to develop the softening process, which will lead to increase in flow stress. The DRX occurs readily in magnesium and its alloys. According to Ion and his coworkers [12], it can be attributed to the constraint imposed by the lack of easily activated slip systems in hexagonal close packed crystal structure, the low stacking fault energy and also the high grain boundary diffusion rate of magnesium.

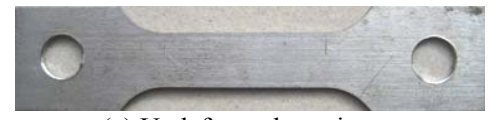

(a) Undeformed specimen

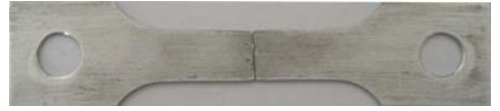

(b) $50{ }^{\circ} \mathrm{C}$

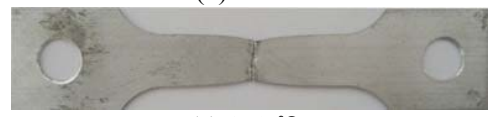

(c) $150{ }^{\circ} \mathrm{C}$

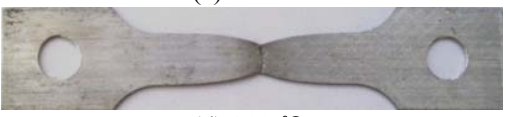

(d) $200{ }^{\circ} \mathrm{C}$

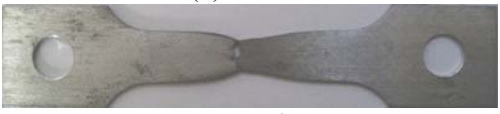

(e) $250{ }^{\circ} \mathrm{C}$

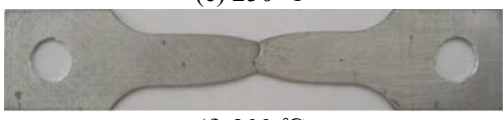

(f) $300{ }^{\circ} \mathrm{C}$

Fig. 3. Contrast before and after uniaxial tensile specimens.

Fig. 5 shows the variation of the elongation at failure with temperature under different strain rates. At $50{ }^{\circ} \mathrm{C}$, the elongation to failure is only $16.7 \%$. As temperature increases, the ductility of AZ31B magnesium alloy is significantly improved. At $250{ }^{\circ} \mathrm{C}$ and a strain rate of $0.1 \mathrm{~s}^{-1}$, the elongation to failure reaches $64.6 \%$. But as temperature continues to rise, the elongation shows a decline trend. At $300^{\circ} \mathrm{C}$ and $0.1 \mathrm{~s}^{-1}$, the elongation to failure is reduced to $60.8 \%$.This can be attributed to grain coarsening and high-temperature oxidation in the deformation process. At a fixed temperature, the elongation of AZ31B magnesium alloy increases with strain rate changing from $0.1 \mathrm{~s}^{-1}$ to $0.001 \mathrm{~s}^{-1}$. The maximum elongation value of $73.3 \%$ was obtained at $250{ }^{\circ} \mathrm{C}$ and a strain rate of $0.001 \mathrm{~s}^{-1}$. 


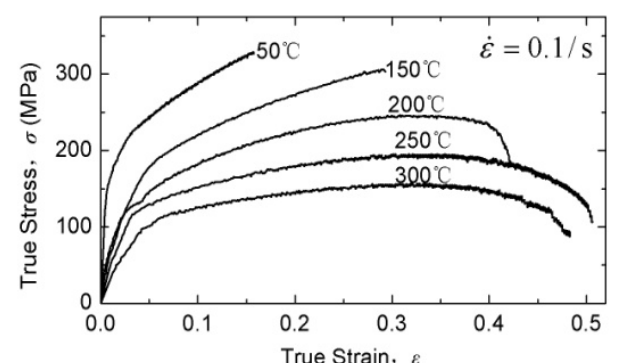

(a)

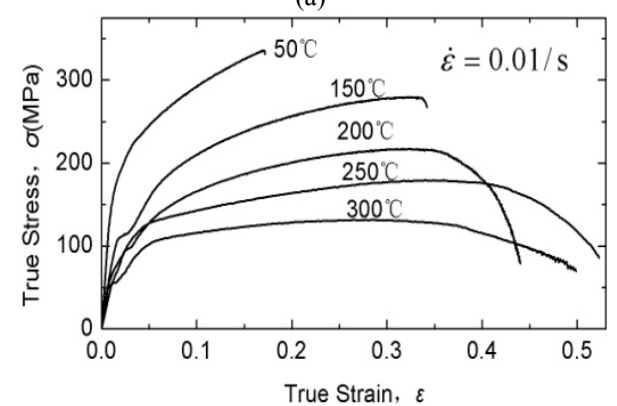

(b)

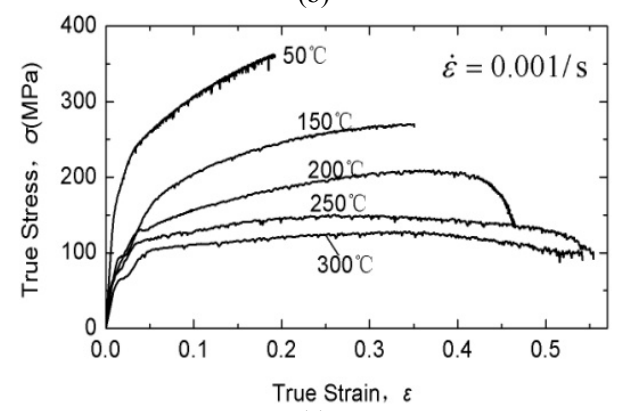

(c)

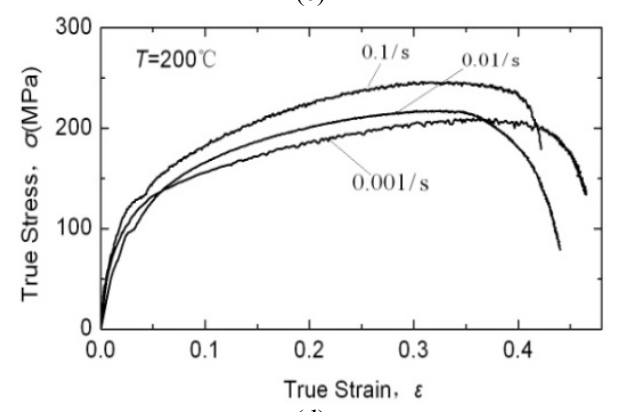

(d)

Fig. 4. True stress-strain curves obtained from the AZ31B sheet tension tests at different temperatures and strain rates.
(a) $\dot{\varepsilon}=0.1 / \mathrm{s}$
(b) $\dot{\mathcal{E}}=0.01 / \mathrm{s}$
(c) $\dot{\varepsilon}=0.001 / \mathrm{s}$
(d) $\mathrm{T}=200{ }^{\circ} \mathrm{C}$

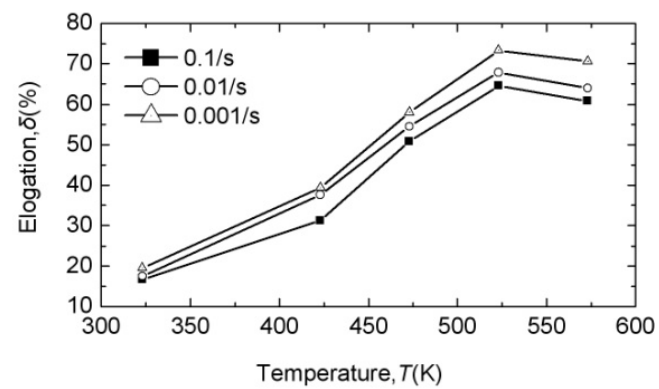

Fig. 5. Relationship between temperature and fracture elongation under different strain rates.

\section{MODEl FOR Flow STRESS ANALysis}

As can be seen in Fig. 4, at low temperatures, the curve exhibits a nearly straight line in the uniform deformation stage. So a double linear can be used to fit the data points collected from the entire deformation process. The mathematical models of flow stress for AZ31B magnesium alloy, as will be mentioned below, only fit to high temperature of more than $150{ }^{\circ} \mathrm{C}$.

In this section, we will concentrate our discussion on two mathematical models: The model based on Fields Backofen equation and the model based on an exponential function.

\section{A. Fields-Backofen Model}

A Fields-Backofen constitutive equation was firstly introduced to describe the flow stress curves of AZ31B magnesium alloy,

$$
\sigma=K \varepsilon^{n} \dot{\varepsilon}^{m}
$$

where $K$ is the strength coefficient, $n$ is the strain- hardening exponent, and $m$ is the strain rate sensitivity exponent. For a fixed temperature and strain rate, the values of $K, n$ and $m$ are all constant. However they will happen to change with changing temperature or strain rate, and therefore need to be modified as a function of temperature and strain rate.

The strain hardening exponents, $n$, at constant temperature and strain rate can be calculated by the following formula:

$$
n=\left.\frac{\partial \lg \sigma}{\partial \lg \varepsilon}\right|_{\dot{\varepsilon}, T}
$$

Fig. 6 shows the relationships between the strainhardening exponents, $n$-value, and the strain rate for various temperatures in semi-log scale. The linear relationships are observed between the $n$-value and the logarithmic of strain rate, namely

$$
n=A+B \lg \dot{\varepsilon}
$$

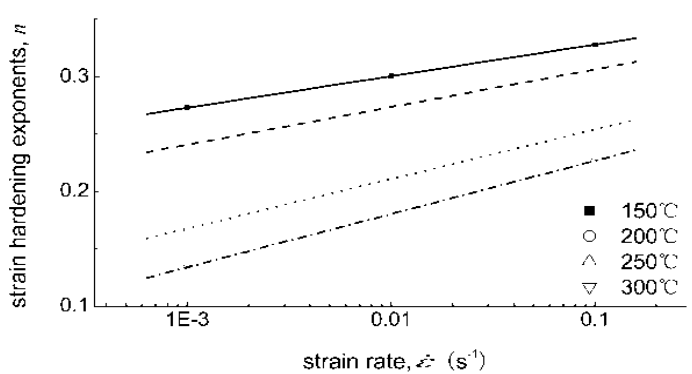

Fig. 6. Relationships between strain hardening exponent and strain rate

The relationship between the parameters $A, B$ and the reciprocal of temperature are showed in Fig. 7, respectively. The linear relationship is found between the parameters $A, B$ and the reciprocal of temperature, and so the $A$-value and $B$-value are approximately given

$$
\begin{aligned}
& \mathrm{A}(\mathrm{T})=0.03482+138.3665 / \mathrm{T} \\
& \mathrm{B}(\mathrm{T})=0.1035-32.4606 / \mathrm{T}
\end{aligned}
$$

Fig. 8 shows the relationships between the flow stress and strain rate at the strain $\varepsilon=0.15$ for various temperatures in $\log -\log$ scale. The linear relationship is also found between 
the logarithmic of flow stress and the logarithmic of strain rate at a certain strain and temperature. The slope of the regression line is equal to the strain rate sensitivity exponent $m$. The strain rate sensitivity coefficient, $m$-value, depends on the temperature. It can be observed in Fig. 9 that the quadratic relationship is found between the $m$-value and the reciprocal of temperature. Therefore, the $m$-value is approximately given by

$\mathrm{m}(\mathrm{T})=-1.5939 \times 105 / \mathrm{T} 2+6.2728 \times 102 / \mathrm{T}-0.5733(6)$

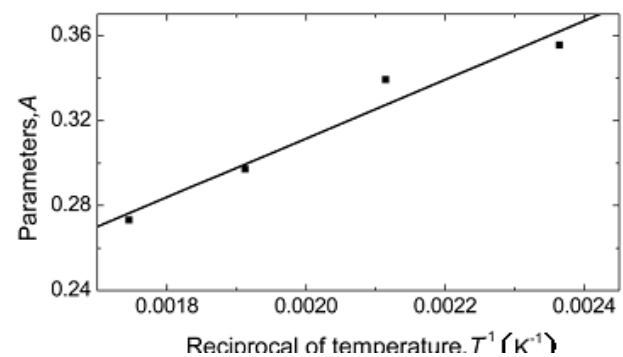

(a)

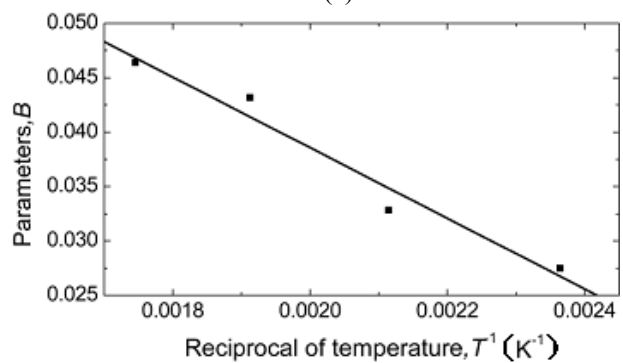

(b)

Fig. 7. Relationships between the parameters A, B and reciprocal of temperature.

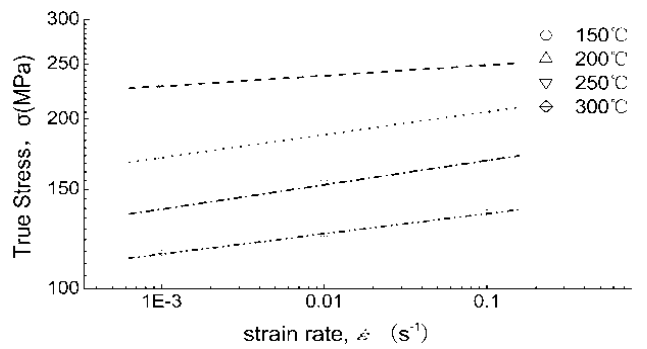

Fig. 8. Relationships between stress and strain rate in log-log scale.

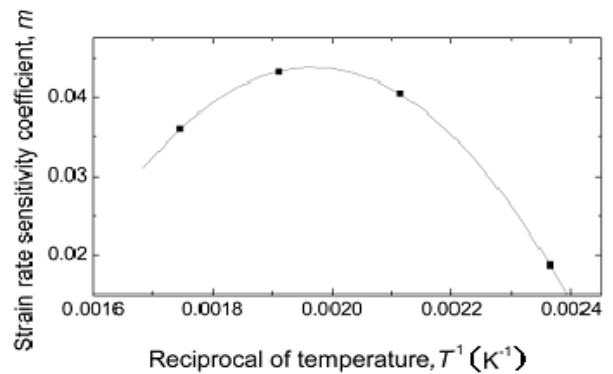

Fig. 9. Relationships between strain rate sensitivity coefficient and reciprocal of temperature.

Fig. 10 shows the relationships between strength coefficient, $K$-value, and strain rate at the strain $\varepsilon=0.15$ for various temperatures in semi-log scale, respectively. The relationship between the $K$-value and the stain rate is similar to that of the n-value. Therefore, the $K$ value can be expressed as:

$$
K=K_{2} \dot{\varepsilon}^{n_{2}}
$$

The values of the parameters $K_{2}$ and $n_{2}$ depend on temperature and can be determined by regression analysis of experimental stress-strain data. These relationships can be expressed in the following equation, respectively

$$
\begin{aligned}
\operatorname{LgK} 2(T) & =-6.83 \times 10-6 \mathrm{~T} 2+0.0047 \mathrm{~T}+1.9187 \\
\mathrm{n} 2(\mathrm{~T}) & =-0.0448+1.5 \times 10-4 \mathrm{~T}
\end{aligned}
$$

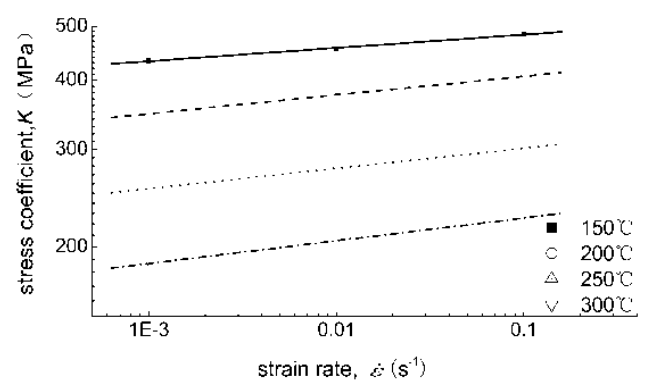

Fig. 10. Relationships between strength coefficient and strain rate in $\log -\log$ scale.

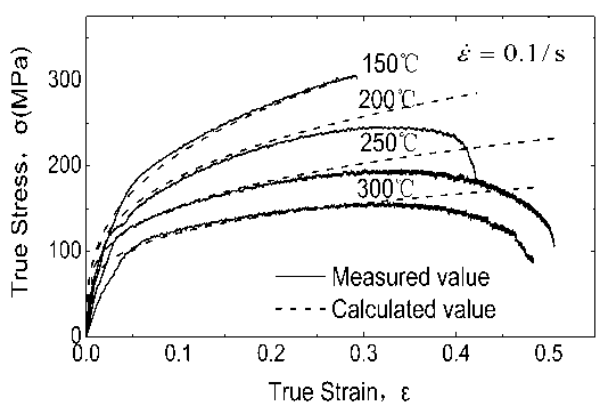

(a)

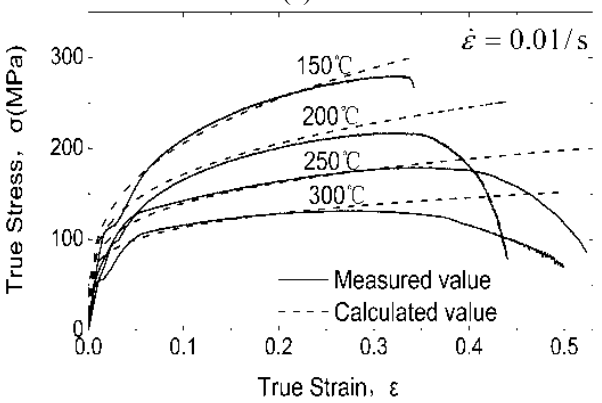

(b)

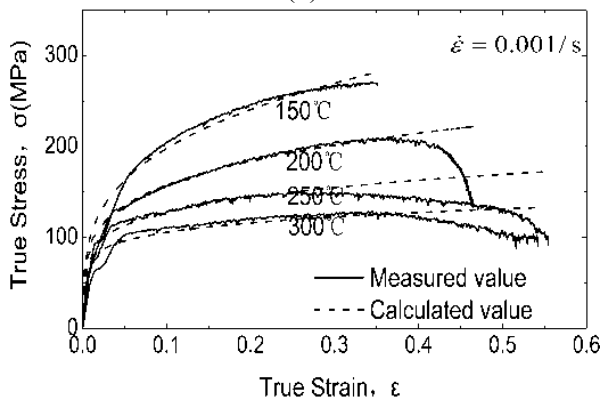

(c)

Fig. 11. Comparison between calculated and measured flow curves.

Fig. 11 shows the comparison between the stress strain curves calculated by the Fields-Backofen model and the experimental data. It can be seen that the calculated flow curves based on Fields-Backofen equation agree well with the measured ones at uniform deformation stage before the peak stress. The lower the temperature, the nearer the trend. As temperature increases, the bifurcation occurs between the predicted curves and experimental ones, which can be 
attributed to the occurrence of dynamic recrystallization at elevated temperatures. So, the model built based on Fields-Backofen equation is inaccurate to describe the softening behavior after the peak stress. For that reason, a new model is proposed in this paper to describe the dynamic softening stage of the flow stress curves for AZ31B magnesium alloy at elevated temperatures.

\section{B. Exponential Model}

Fig. 12 shows the relationships between true stress and true strain at a strain rate of $0.01 \mathrm{~s}^{-1}$ for various temperatures in semi-log coordinate system. Neglecting the low strain range under 0.05 and the high strain range after necking, a quadratic relationship is observed between the nature logarithmic of true stress and true strain for AZ31 AZ31B magnesium alloy, namely

$$
\sigma=e^{a+b \varepsilon+c \varepsilon^{2}}
$$

The parameters $a, b$ and $c$ in the equation (10) are not constant but change with temperature and strain rate. That is, under a fixed temperature and strain rate, they are all constant, whereas they will happen to change with changing temperature and strain rate. So, the parameters $a, b$ and $c$ need be amended based on the experiment results at various temperatures and strain rates.

Fig. 13 shows the relationship between the parameters a, $\mathrm{b}$ and $\mathrm{c}$ and temperature at a strain rate of $0.01 \mathrm{~s}-1$.Obviously, under a constant strain rate, the linear relationship can be observed between the parameters $a, b, c$ and temperature, that is

$$
\begin{aligned}
& a=X_{1}+X_{2} T \\
& b=X_{3}+X_{4} T \\
& c=X_{5}+X_{6} T
\end{aligned}
$$

Linear regression is made on experimental data to get the parameters $X_{1} \sim X_{6}$ in the equation (12) - (13) under various strain rates respectively. As can be seen from Fig. 14, the parameters $X_{1} \sim X_{6}$ are almost linear with the logarithm of strain rate at fixed temperature. Namely,

$$
X_{i}=A_{i}+B_{i} \lg \dot{\varepsilon} \quad(i=1,2, \ldots, 6)
$$

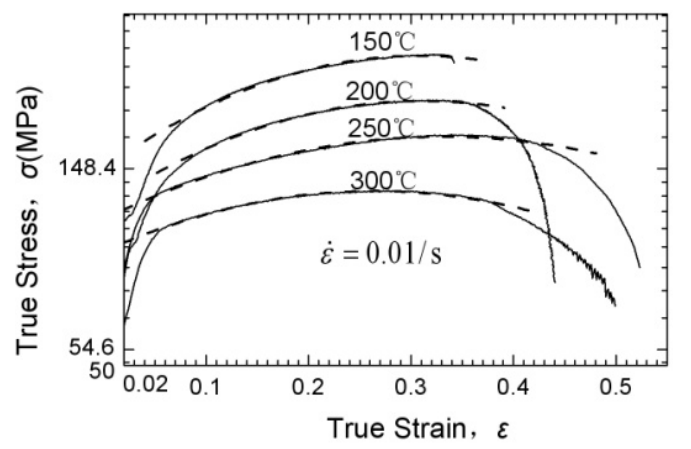

Fig. 12. Relationship between nature logarithmic of true stress and true strain.

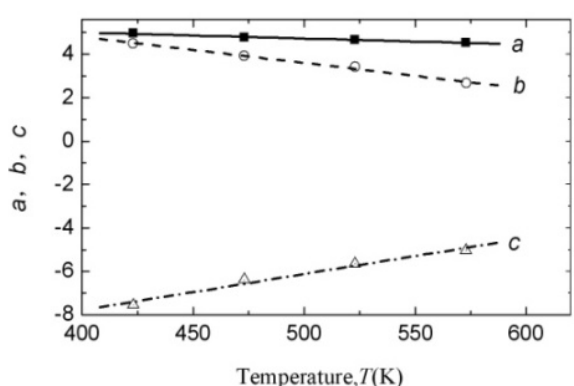

Fig. 13. Relationship between parameters a,b,c and temperature.

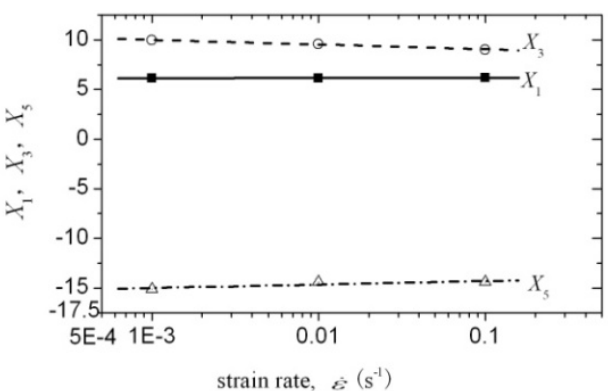

(a)

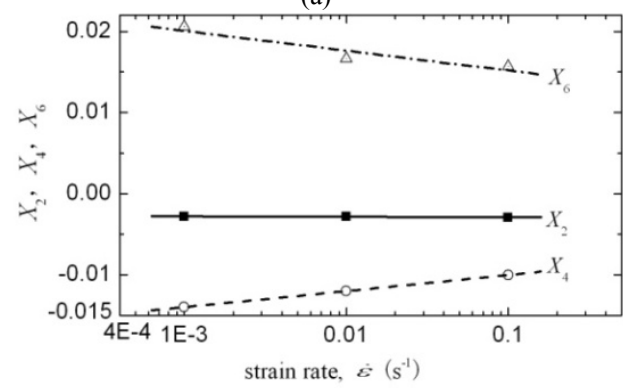

(b)

Fig. 14. Relationship between parameters X1 X6 and nature logarithmic of strain rate.

TABLE I: THE COEFFICIENT AND THE FITTING RESULTS IN THE EQUATION (14)

\begin{tabular}{ccc}
\hline \hline$i$ & $A_{i}$ & $B_{i}$ \\
\hline 1 & 6.22943 & 0.03892 \\
2 & -0.00292 & -0.00004 \\
3 & 8.57906 & -0.47297 \\
4 & -0.00801 & 0.00199 \\
5 & -13.9373 & 0.3525 \\
6 & 0.01274 & -0.00245 \\
\hline \hline
\end{tabular}

Through the linear regression, the parameters $A_{i}$ and $B_{i}$ are obtained and listed in table1. Substituting the corresponding parameters $A_{i}, B_{i}$ and strain rate into the equation (14), the parameters $X_{1} \sim X_{6}$ under different strain rates can be obtained. And then substituting the parameters $X_{1} \sim X_{6}$ and the corresponding temperature into the equation (11) - (13) respectively, the parameters $a, b$ and $c$ in the equation (10) can be determined under a certain temperature and strain rate. Eventually substituting $a, b$ and $c$ value into the formula (10), the formula of the corresponding flow stress would be determined.

Fig. 15 shows the comparison between the stress-strain curves calculated by the exponential model and the experimental data. The calculated flow curves agree well with the measured ones at uniform deformation stage. After the peak stress the gap between the predicted values and 
experimental data increases gradually, but they still keep consistent trend. At higher strain rates, the flow curves calculated by this model have better coincidence with the measured ones; whereas as strain rate decreases, the difference tends to increase. Considering the actual forming process of magnesium alloys, lower strain rates (such as $0.001 \mathrm{~s}^{-1}$ ) have no practical significance and are usually ignored. Compared with the Fields-Backofen model, the exponential model can accurately reflect the work hardening and strain softening effect of AZ31B magnesium alloy sheet at high temperatures. Therefore, the second model built in this paper is more appropriate for the prediction of flow stress of AZ31B magnesium alloys at high temperatures.

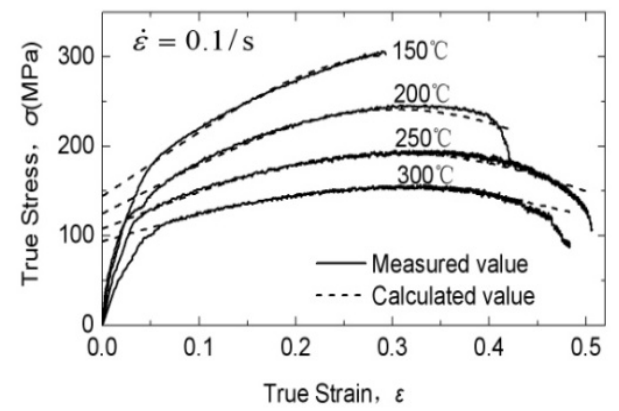

(a)

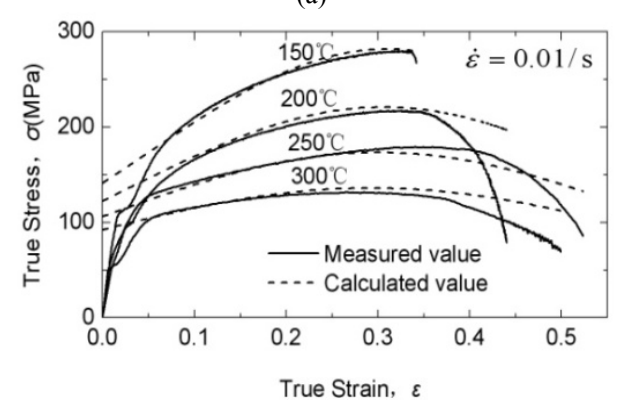

(b)

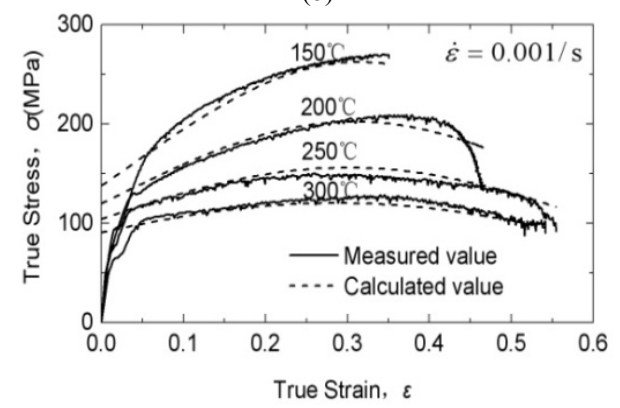

(c)

Fig. 15. Comparison between calculated and measured flow curves.

\section{CONCLUSIONS}

In this study, the true stress-strain curves of AZ31B magnesium alloy obtained by uniaxial tensile tests at various conditions were analyzed, the main conclusions are as follows.

1) An obvious softening phenomenon was observed during hot tensile deformation of AZ31B magnesium alloy sheet. And as the temperature increase and/or strain rate decreases, the softening effect is more notable. The peak stress decrease and elongation-to-failure increase with the increase in temperature and the decrease in strain rate, the formability can be significantly improved.

2) Two mathematical models were developed to determine flow stress at hot deformation conditions:(1) the model based on the Fields-Backofen equation, and (2) the model based on a natural exponential function whose exponent is a quadratic function. By comparison of calculated results with experimental data, it has been proved that the second model can well reflect work hardening and strain softening effect of AZ31B magnesium alloy during hot tensile deformation, and thus is more appropriate for the prediction of flow stress for AZ31B magnesium alloys at high temperatures and the numerical simulation of forming processes for AZ31B magnesium alloy sheet.

\section{ACKNOWLEDGMENT}

The authors would like to acknowledge the financial support from the National Natural Science Foundation of China under grant No. 50905098, and Program for Changjiang Scholars and Innovative Research Team in University of Ministry of Education of China under grant No. IRT0931.

\section{REFERENCES}

[1] B. L. MORDIKE and T. EBERT, "Magnesium:Properties-applications-potential," Materials Science and Engineering, vol.302, no.5, pp. 37-45, April 2001.

[2] H. Somekawa and T. Mukai, "Effect of grain refinement on fracture toughness in extruded pure magnesium," Scripta Materialia, vol. 53, no. 9, pp.1059-1064, Nov. 2005

[3] Z. YANG, J. P. LI, J. X. ZHANG, G. W. LORIMER, and J. ROBSON, "Review on research and development of magnesium alloys," Acta Metallurgica Sinica (English Letters), vol. 21, no.5, pp.313-328, Oct.2008.

[4] E. DOEGE and K. DRODER, "Sheet metal forming of magnesium wrought alloys-formability and process technology," Journal of Materials Processing Technology, vol. 115, no.1, pp. 14-19, Aug.2001

[5] Z. Gao, "Microstructural stability of magnesium alloys during high temperature deformation," M.S. thesis, Dept. Mat. Eng., McMaster Univ., Hamilton, Canada,

[6] F. K. Chen and T. B. Huang, "Formability of stamping magnesium-alloy AZ31 sheets," Journal of Materials Processing Technology, vol. 142, no. 3, pp. 643-647, Dec. 2003.

[7] Y. Q. Cheng, H. Zhang, Z. H. Chen, and K. F. Xian, "Flow stress equation of AZ31 magnesium alloy sheet during warm tensile deformation," Journal of materials processing technology, vol. 208, no.1-3, pp. 29-34, Nov. 2008

[8] J. Liu, Z. S. Cui, and C. X. Li, "Modeling of flow stress characterizing dynamic recrystallization for magnesium alloy AZ31B," Computational Materials Science, vol. 41, no.3, pp.375-382, Jan.2008.

[9] H. Takuda, H. Fujimoto, and N. Hatta, "Modeling on flow stress of Mg-Al-Zn alloys at elevated temperatures," Journal of Materials Processing Technology, vol. 80-81, pp. 513-516, Aug.1998.

[10] Z. Q. Sheng and R. Shivpuri, "Modeling flow stress of magnesium alloys at elevated temperature," Materials Science and Engineering, vol. 419, no.1-2, p p. 202-208, March 2006

[11] X. H. Zhang, "Experimental and numerical study of magnesium alloy during hot-working process," Ph.D. dissertation, sch. Mat. Eng., Shanghai Jiao tong Univ., Shanghai, China, 2003.(in Chinese)

[12] S. E. Ion, F. J. Humphreys, and S. H. White, "Dynamic recrystallisation and the development of microstructure during the high temperature deformation of magnesium," Acta Mater, vol. 30, no.10, pp.1909-1919, Oct.1982.

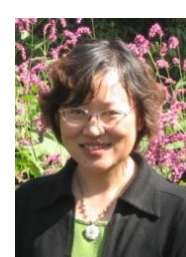

LI Wen-juan Date of birth: July 4, 1971, Education: Ph.D Candidate, Materials processing engineering, School of Materials Science and Engineering, Shandong University, China. The main research field: stamping process and deformation mechanism of magnesium alloy sheet and numerical simulation Telephone: +86 531 88393238 Fax: +86 53188392811 Mobile phone: 15666018696, e-mail: liwj@sdu.edu. 